\title{
Successful Non-Surgical Treatment of Brain Abscess and Necrotizing Fasciitis Caused by Bacillus cereus
}

\author{
Takehiko Mori, Michihide TokuhIRA*, Yujiro TAKaE**, Shigehisa Mori*, \\ Hirosi SuZuKI***, Toru ABE* and Tsutomu TAKEUCHI*
}

\begin{abstract}
Musculoskeletal and central nervous system infections caused by Bacillus cereus are very rare. Only a few cases have been reported, whose clinical courses strongly suggested that surgical procedures combined with appropriate antimicrobial therapy are necessary to cure these infections. A 60-year-old man with severe neutropenia due to myelodysplastic syndrome, developing necrotizing fasciitis and brain abscess caused by Bacillus cereus is reported. Without performing any surgical procedures, the patient was successfully treated with systemic antimicrobial therapy combined with granulocyte colony stimulating factor, which contributed to the increase in the neutrophil count.
\end{abstract}

(Internal Medicine 41: 671-673, 2002)

Key words: myelodysplastic syndrome, neutropenia, granulocyte-colony stimulating factor

\section{Introduction}

Bacillus species other than Bacillus anthracis had generally been discarded as laboratory contaminants. However, these organisms, particularly Bacillus cereus, have been increasingly recognized to cause serious human infections, mainly in immunocompromised hosts (1-3). We herein report a case with myelodysplastic syndrome, developing necrotizing fasciitis and brain abscess caused by Bacillus cereus. Because of severe thrombocytopenia, surgical procedures that were generally considered necessary for the treatment of these severe infections (3-7) were not performed. However, the patient was successfully treated with systemic antimicrobial therapy combined with granulocyte-colony stimulating factor (G-CSF).

\section{Case Report}

A 60-year-old Japanese man was diagnosed as having myelodysplastic syndrome. At diagnosis, blood examination revealed a severe pancytopenia with less than $100 / \mu 1$ of neutrophils. Two months after the diagnosis, he was admitted to the hospital with a fever of $39.5^{\circ} \mathrm{C}$ and swelling of the right lower leg. The swelling extended to the femur and lower abdominal wall within a week. Computed tomography of the right femur and lower leg demonstrated intramuscular and subcutaneous low density lesions that were compatible with necrotizing fasciitis and cellulitis (Fig. 1). Blood examinations revealed a leukocyte count of $700 / \mu 1$ without any neutrophils, and creatine phosphokinase of $687 \mathrm{IU} / l$ (normal range: less than 170 $\mathrm{IU} / l)$. On the fifth hospital day, he became increasingly disoriented. Magnetic resonance imaging (MRI) of the brain demonstrated a mass lesion at the left thalamus with ring enhancement by gadolinium, compatible with the brain abscess (Fig. 2). Bacillus cereus was isolated from the blood on four occasions. It was susceptible to gentamicin, vancomycin, panipenem, clindamycin, ciprofloxacin, clarithromycin, and minocycline, and resistant to penicillins and cephalosporins by the disk diffusion method. Other pathogens were not isolated. Because of the severe thrombocytopenia (less than $1.5 \times 10^{4} / \mu 1$ ), surgical procedures, including excision, debridement, and amputation, were not indicated. The antimicrobial regimen with gentamicin (120 mg daily i.v.), panipenem (2 g daily i.v.), clindamicin (1,200 mg daily i.v.), and ciprofloxacin $(600 \mathrm{mg}$ daily oral) were initiated. In addition, G-CSF was also administered for neutropenia, and his neutrophil count increased to $800 / \mu 1$. Two days after initiating this combination therapy, Bacillus cereus was no longer isolated from the blood. The swelling of the right extremity and neurological symptoms began to improve. After being treated with the combination therapy for 50 days, CT and MRI demonstrated complete improvement of the lesions in both the right leg and the left thalamus. No adverse effects other than allergic reaction to

From the Division of Hematology, Department of Medicine, Keio University School of Medicine, Tokyo, *Second Department of Internal Medicine, **Department of Dermatology, Saitama Medical Center, Saitama Medical School, Kawagoe and ${ }^{* * * T r a n s f u s i o n ~ C e n t e r, ~ N a t i o n a l ~ D e f e n s e ~ M e d i c a l ~ C o l l e g e, ~}$ Tokorozawa

Received for publication November 28, 2001; Accepted for publication April 5, 2002

Reprint requests should be addressed to Dr. Takehiko Mori, the Division of Hematology, Department of Medicine, Keio University School of Medicine, 35 Shinanomachi, Shinjuku-ku, Tokyo 160-8582 




Figure 1. Necrotizing fasciitis and cellulitis of the right lower leg. Computed tomography demonstrated subcutaneous and intramascular low density lesions.

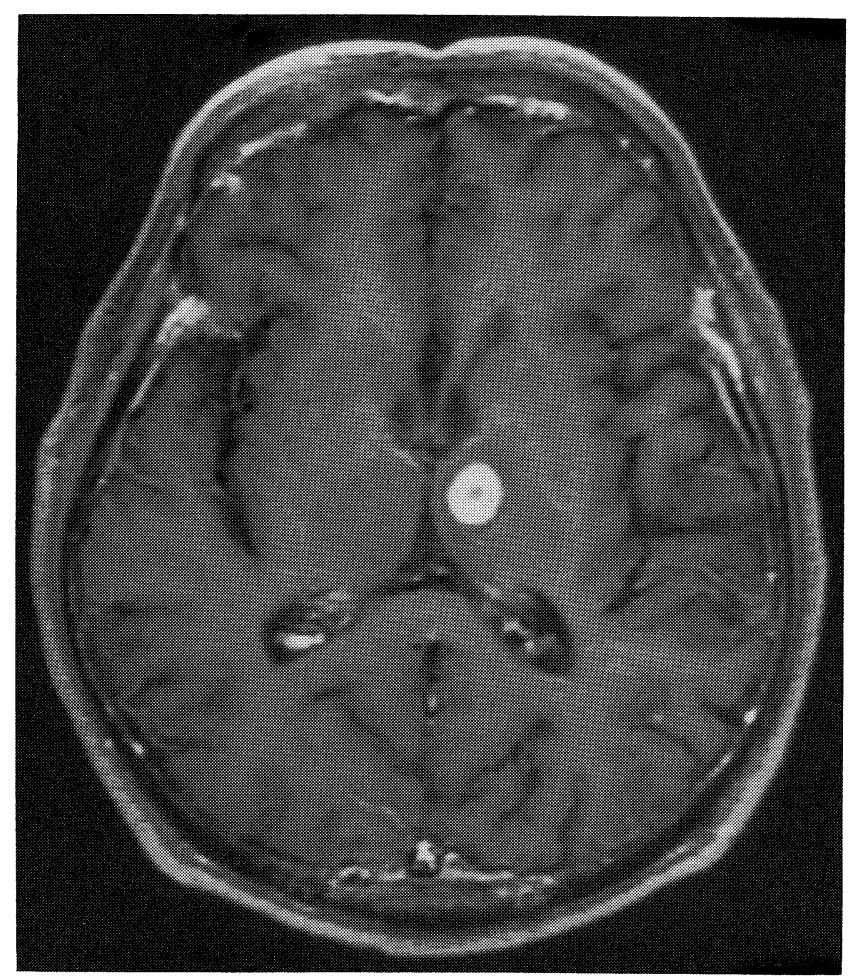

Figure 2. Brain abscess caused by Bacillus cereus. Magnetic resonance imaging (MRI) of the brain demonstrated a mass lesion at the left thalamus with ring enhancement by gadolinium. panipenem were observed. When the patient was discharged 10 weeks after the onset, he was free from symptoms except for a mild memory deficit.

\section{Discussion}

Bacillus species other than Bacillus anthracis, particularly Bacillus cereus, have been recognized as the pathogens of a variety of human diseases, including food poisoning, pneumonia, endophthalmitis, and musculoskeletal and central nervous system infections (1-3). Although culture samples were not directly obtained from the affected organs, the brain and the lower extremity, we determined Bacillus cereus as the causative pathogen, because the blood cultures were repeatedly positive for this pathogen, and the clinical manifestations rapidly improved after blood cultures became negative. To the best of our knowledge, only 7 cases of brain abscess caused by Bacillus species have been reported in the English language literature (7-11). Five of the 7 cases succumbed due to the infection. The previous reports have shown the efficacy and necessity of surgical procedures for brain abscess and musculoskeletal infections due to Bacillus species, although these surgical procedures often result in severe organ damage or deficit. Here, although surgical procedures were considered, they could not be performed in the present case because of severe thrombocytopenia. Also, the existence of two infectious sites was another reason. However, disease was eventually cured with long-term combination antimicrobial therapy, thus resulting in no organ damage or deficit due to surgical procedures. In addition, the administration of G-CSF which induced the increase in neutrophil counts could also contribute to the resolution of the disease (7).

In conclusion, the findings of the present case suggest that Bacillus cereus infections such as brain abscess and necrotizing fasciitis might be treatable by systemic antimicrobial therapy alone in certain patients if the predisposing condition such as neutropenia can be improved.

\section{References}

1) Tuazon CU. Other Bacillus species. in: Principles and Practices of Infectious Diseases. 4th ed. Mandell GL, Bennett JE, Dolin R, Eds. Churchill Livingstone, New York, 1995: 1890-1894.

2) Banerjee C, Bustamante CI, Wharton R, Talley E, Wade JC. Bacillus infections in patients with cancer. Arch Intern Med 148: 1769-1774, 1988.

3) Sliman R, Rehm S, Shlaes DM. Serious infections caused by Bacillus species. Medicine (Baltimore) 66: 218-223, 1987.

4) Tuazon CU, Murray HW, Levy C, Solny MN, Curtin JA, Sheagren JN. Serious infections from Bacillus sp. JAMA 241: 1137-1140, 1979.

5) Kemmerly SA, Pankey GA. Oral ciprofloxacin therapy for Bacillus cereus wound infection and bacteremia. Clin Infect Dis 16: 189, 1993.

6) Ihde DC, Armstrong D. Clinical spectrum of infection due to Bacillus species. Am J Med 55: 839-845, 1973.

7) Sakai C, Iuchi T, Ishii A, Kumagai K, Takagi T. Bacillus cereus brain abscesses occurring in a severely neutropenic patient: Successful treatment with antimicrobial agents, granulocyte-colony stimulating factor and surgical drainage. Int Med 40: 654-657, 2001.

8) Pennington JE, Gibbons ND, Strobeck JE, Simpson GI, Myerowitz RI. Bacillus species infection in patients with hematologic neoplasia. JAMA 


\section{Treatment of Bacillus cereus Infection}

235: 1473-1474, 1976.

9) Jenson HB, Levy SR, Duncan C, Mcintosh S. Treatment of multiple brain abscess caused by Bacillus cereus. Pediatr Infect Dis J 8: 795-798, 1989.

10) Jones BL, Hanson MF, Logan NA. Isolation of Bacillus licheniformis from a brain abscess following a penetrating orbital injury. J Infect 24:
103-104, 1992.

11) Bert F, Ouahes O, Lambert-Zechovsky N. Brain abscess due to Bacillus macerans following a penetrating periorbital injury. J Clin Microbiol 33: 1950-1953, 1995. 